\title{
Martínez Baztán, Alfonso. (2011) La evaluación de las lenguas. Ga- rantías y limitaciones. Andalucía: Editorial Octaedro.
}

Martha Jurado Salinas

CEPE-UNAM

Durante los últimos 25 años ha ido haciéndose cada vez más evidente la necesidad de contar con sistemas de evaluación que ofrezcan garantías sobre sus métodos y objetivos, y que a la vez tengan en cuenta las características y necesidades de los candidatos. Otros fenómenos relacionados han tenido también una rápida evolución: la publicación de libros divulgativos en inglés y otras lenguas sobre las técnicas de diseño de test, la diversificación de contextos de evaluación, así como los tipos de candidatos y los formatos y habilidades que se necesitan medir. Todo esto ha hecho patente la necesidad de entender el sentido y funcionamiento de los exámenes para poder diseñarlos de acuerdo a diversas necesidades, según los diferentes contextos en que se producen y las distintas y variadas personas que los usan o requieren.

La obra de Alfonso Martínez Baztán, La evaluación de las lenguas: garantías y limitaciones, cubre estos objetivos. Por una parte, tiene carácter informativo, pero también educativo y formativo; por otra, sirve de ayuda a todos aquellos involucrados en estas crecientes necesidades, que no son profesionales de la evaluación: personal relacionado con la enseñanza de lenguas (profesores de español para extranjeros o de otras lenguas), así como personas que tienen que planificar, diseñar exámenes (para fines educativos o académicos como sociales, laborales u otros) y emitir notas, haciendo explícitos sus criterios de calificación, sus objetivos y formatos.

El libro presenta una dificultad de nivel medio, apta para los no profesionales de la evaluación. No se trata de una publicación básica que vuelve a explicar sobre los exámenes y sus tipologías, sobre las tareas e ítems (reactivos) y sus características, modos de diseño, ventajas e inconvenientes, pero tampoco entra en consideraciones demasiado técnicas o en explicaciones estadísticas complejas. Intenta ayudarnos a entender los mecanismos de los exámenes y a hacer con ellos test lo más profesionalmente posible. Pero sobre todo, trata de hacernos conscientes de que la evaluación es una tarea que necesita dotarse de herramientas adecuadas que prueben que las mediciones que se realizan con ellas son objetivas, válidas y fiables. 
Para ello, uno de los primeros objetivos del libro es mostrar cómo la evaluación debe y puede realizarse con garantías. En este sentido el texto puede ser interesante al menos por tres razones:

- ofrece una explicación clara de cómo funcionan los test explicando su verdadero alcance y posibilidades, haciendo saber que no es posible medir todo lo que se desee o planifique sino solo aquello que efectivamente se haya logrado alcanzar mediante las herramientas diseñadas;

- presenta información práctica —basada en los numerosos talleres impartidos por su autor y las dudas y preguntas que en ellos se plantean- sobre cómo funcionan los ítems y test, y sobre las dificultades que presenta el diseño de exámenes fiables y válidos de manera que sirvan a nuestros deseos y no nos lleven a realizar juicios erróneos o inapropiados sobre las habilidades de los candidatos;

- toma como base el Marco Común Europeo de Referencia (MCER), tanto para mostrar su uso, en la media de lo posible, como para hacer ver sus dificultades de aplicación o sus limitaciones.

En él se explican con realismo las posibilidades y limitaciones que se van a encontrar y se señala claramente el verdadero alcance de las herramientas ya sean innovadoras o previamente existentes. En la medida en que dispongamos de equipos técnicos y profesionales que diseñen herramientas fiables, y de más personas para llevar a cabo una buena y objetiva experimentación, se podrán alcanzar estándares de validez y garantías similares a las de un test aplicado a un gran número de candidatos de edades, culturas y procedencia distintas, con lo cual se asegura que todos ellos tienen las mismas oportunidades y funciona y se adecua a todos por igual, sin premiar a unos en detrimento de otros. Pero si nuestras necesidades son más limitadas y nuestras posibilidades u objetivos más sencillos, como en la mayoría de centros de enseñanza, centros de lenguas, escuelas, academias o centros universitarios, debemos comenzar por conocer y cotejar la posibilidad de alcanzar nuestros objetivos con los materiales técnicos y los equipos humanos con que contemos. Así podremos planear y obtener de nuestros exámenes el máximo rendimiento, sin despreciar ni desconocer sus limitaciones, y evitando extraer conclusiones falsas, subjetivas o irreales.

El libro presenta una introducción, ocho capítulos, un epílogo, cuatro apéndices y una amplia y actualizada bibliografía sobre el tema.

El capítulo 1 funciona a modo de introducción advirtiendo de los objetivos anteriormente mencionados. Señala los cambios acaecidos en los últimos años, incluida la publicación del MCER, y la necesidad de proceder a homologaciones entre niveles y pruebas demostrando que los resultados son inteligibles y se 
pueden comparar entre diversos países, niveles y lenguas. Todo esto supone una nueva responsabilidad para la profesión de los profesores de lengua; una responsabilidad que se suma a las ya muchas que se concentran en este campo de trabajo cada vez más importante y decisivo como es el aprendizaje de lenguas extranjeras. Responsabilidad, se advierte, necesitada de grupos de trabajo y equipos especializados y profesionalizados, aunque — como se apunta en el capítulo 2-comandados por los profesores, los primeros interesados, implicados y conocedores de la materia a tratar: la lengua de sus estudiantes.

Los capítulos 2 y 3, Garantías y limitaciones de la evaluación de lenguas y Herramientas de evaluación, se centran en una explícita defensa de la necesidad de dotar de garantías a todo el proceso de diseño y evaluación, y explican varias de las herramientas generalmente usadas en la evaluación de lenguas, como las escalas, las especificaciones o las notas, señalando su sentido y utilidad así como sus limitaciones. Son explicaciones que cobran mayor sentido y funcionalidad gracias a la nueva luz que arrojan sobre elementos de la evaluación que por su familiaridad se suelen descuidar provocando errores de medición. Son interesantes, por ejemplo, los apartados dedicados a comprender el sentido de las notas para poder elaborar con ellas los juicios que se pretenden respecto a las habilidades de los candidatos; a la explicación de las diferentes escalas y las consecuencias de su uso en los diferentes modelos de evaluación; o al análisis de la subjetividad de ciertos elementos de los test y su apuesta por delimitarla y cercarla al máximo para ver sus consecuencias y minimizar sus efectos en lo posible. Los elementos subjetivos se deben identificar y si no se pueden hacer más objetivos, se deben eliminar y aceptar que el test planeado no ha podido ser diseñado hasta sus últimas consecuencias y que lo que queda es un test con un alcance menor al esperado.

Los capítulos 4 y 5, Validación de ítems y Validación de exámenes, describen y dan ejemplos variados del funcionamiento de los ítems y de las tareas, y enseñan cómo deben ser diseñados para que cumplan su función de encontrar a los candidatos que efectivamente conocen o usan mejor la lengua. Muestran los mecanismos que permiten comprobar que un ítem o un test es efectivamente lo que se pretendía al elaborarlo o —en su defecto- averiguar sus limitaciones; porque puede suceder que sean mejor respondidos por candidatos que saben poco, mientras que otros de más nivel pueden verse inducidos a cometer errores, situación que distorsiona la evaluación y sus objetivos. Interesantes resultan las formas de averiguar la dificultad de un ítem o la medida de su funcionamiento efectivo, dos datos necesarios para seleccionar los apropiados para componer un buen examen para un nivel determinado. El capítulo 5 se centra en los exámenes, - una vez compuestos con buenas tareas y buenos ítems- y en averiguar para qué sirven, en qué medida y cuál es su alcance. Para ello se muestra cómo se puede 
analizar información para determinar si dos test similares miden lo mismo (no siempre es ni tiene por qué ser así) o si dos test diferentes miden habilidades o contenidos realmente diferentes (algo que no es tan evidente como parece). La explicación de las correlaciones entre tests o partes de un examen -la comparación de sus efectos y resultados- aclara de forma sencilla estos aspectos.

Los capítulos 6 al 9 dividen los tipos de test existentes según su uso, sea académico, capítulos 6 y 7; o social, capítulos 8 y 9. Ambos capítulos explican brevemente algunos aspectos interesantes sobre cada tipo de pruebas y las características de su diseño.

El capítulo 6 se centra específicamente en la explicación de las características y peculiaridades de las pruebas de clasificación, útiles y necesarias en todo centro de lenguas para agrupar coherentemente a los estudiantes según sus conocimientos y necesidades y las programaciones y capacidad del centro. Se dan útiles consejos sobre su forma de diseño y —atendiendo a las necesidades de su validaciónse ejemplifica gran parte de lo explicado en el capítulo anterior, haciendo ver que no son conceptos abstractos sino útiles, importantes y necesarios.

El capítulo 7 señala los principales aspectos de las pruebas de progreso, aprovechamiento y diagnóstico.

El capítulo 8 se centra ampliamente en la expresión oral. Presenta distintos enfoques de la evaluación oral con consecuencias diferentes en cuanto al lenguaje elicitado y a las conclusiones que se pueden extraer. El objetivo de este capítulo está en que los diseñadores y evaluadores sean conscientes de que la expresión oral (al igual que las tiene la expresión escrita o las habilidades de compresión) es una destreza con características específicas, entre las cuales se encuentran el vocabulario o la gramática. El autor hace énfasis en que medir y evaluar las habilidades de expresión es más que medir el conocimiento y uso de estos dos elementos formales. Entre sus rasgos distintivos está el hecho de que el examen se materializa mediante la acción de los interlocutores (muchas veces el entrevistador-profesor y el candidato), razón por la cual el entrevistador es corresponsable del nivel surgido/mostrado en la entrevista/examen oral por el candidato. Por este motivo, es muy importante analizar y ser consciente del papel del entrevistador en la génesis del discurso. El capítulo también explica un interesante y fundamental concepto: "el estrés comunicativo". Se defiende la necesidad de identificarlo, pues de lo contrario puede ser contraproducente, engañoso y provocar que el nivel de expresión oral de un candidato parezca menor al que en realidad posee, con lo cual se afectaría negativamente su nota. Se explica el concepto, se delimitan sus causas (la propia estructura del examen, el comportamiento del elicitador, la personalidad del candidato, entre otras) y se intenta que los entrevistadores sean conscientes de este mecanismo de la expresión oral, para — una vez entendido, identificado y captado cuando surjaentender su efecto y solucionarlo o restarlo a la hora de tomar decisiones. 
El capítulo 9 señala los principales aspectos de los exámenes de expresión escrita y comprensión auditiva y lectora.

En fin, esta obra de Martínez Baztán trata de ser una contribución a la necesidad creciente de diseñar exámenes de idiomas y acreditarlos como válidos. Los lectores que estén interesados en planear exámenes y justificar y explicar adecuadamente sus resultados tienen aquí una valiosa información sobre cómo proceder, qué pueden esperar de su trabajo y qué hacer si aspiran a mejorar los resultados. $Y$ también puede ser interesante para aquellos otros que solamente estén parcialmente envueltos en estos procesos y quieran saber más acerca de la situación actual de este tema que ha cobrado tanta importancia en los últimos años. 
